\title{
Structure and function of ALG-2, a penta-EF-hand calcium-dependent adaptor protein
}

\author{
Masatoshi MAKI $^{1 *}$, Hironori SUZUKI ${ }^{1,2} \&$ Hideki SHIBATA ${ }^{1}$ \\ ${ }^{1}$ Department of Applied Molecular Biosciences, Graduate School of Bioagricultural Sciences, Nagoya University, Nagoya 464-8601, Japan; \\ ${ }^{2}$ Structural Biology Research Center, Photon Factory, Institute of Materials Structure Science, High Energy Accelerator Research Organization, \\ Ibaraki 305-0801, Japan
}

Received May 11, 2011; accepted June 5, 2011

\begin{abstract}
ALG-2 (a gene product of PDCD6) is a 22-kD protein containing five serially repetitive EF-hand structures and belongs to the penta-EF-hand (PEF) family, including the subunits of typical calpains. ALG-2 is the most conserved protein among the PEF family members and its homologs are widely found in eukaryotes. X-ray crystal structures of various PEF proteins including ALG-2 have common features: presence of eight $\alpha$-helices and dimer formation via paired EF5s that are positioned in anti-parallel orientation. ALG-2 forms a homodimer and a heterodimer with its closest paralog peflin. Like calmodulin, a well-known four-EF-hand protein, ALG-2 interacts with various proteins in a $\mathrm{Ca}^{2+}$-dependent fashion, but the binding motifs are completely different. With some exceptions, ALG-2-interacting proteins commonly contain Pro-rich regions, and ALG-2 recognizes at least two distinct Pro-containing motifs: $\mathrm{PPYP}(X) n \mathrm{YP}(X$, variable; $n=4$ in ALIX and PLSCR3) and PXPGF (represented by Sec31A). A shorter alternatively spliced isoform, lacking two residues and designated ALG- $2^{\Delta \mathrm{GF} 122}$, does not bind ALIX but maintains binding capacity to Sec31A. X-ray crystal structural analyses have revealed that binding of calcium ions induces the configuration of the side chain of R125 so that it opens Pocket 1, which accepts PPYP, but Pocket 1 remains closed in the case of ALG-2 $2^{\Delta \mathrm{GF} 122}$. ALG-2 dimer has two ligand-binding sites, each in a monomer molecule, and appears to function as a $\mathrm{Ca}^{2+}$-dependent adaptor protein to either stabilize a preformed complex or to bridge two proteins on scaffolds in systems of the endosomal sorting complex required for transport (ESCRT) and ER-to-Golgi transport.
\end{abstract}

\section{ALG-2, calcium, penta-EF-hand, X-ray crystal structure, protein-protein interaction, membrane trafficking}

Citation: Maki M, Suzuki H, Shibata H. Structure and function of ALG-2, a penta-EF-hand calcium-dependent adaptor protein. Sci China Life Sci, 2011, 54: 770-779, doi: 10.1007/s11427-011-4204-8

\section{Introduction: penta-EF-hand (PEF) family}

\subsection{Discovery of PEF domain in calpain}

Calpains are non-lysosomal intracellular cysteine proteinases. Typical calpains such as $\mu$-calpain (calpain-1) and $\mathrm{m}$-calpain (calpain-2) are ubiquitously expressed in mammalian tissues and are activated by micromolar and millimolar levels of $\mathrm{Ca}^{2+}$, respectively, in in vitro protease assays. Molecular cloning of cDNAs for the large catalytic subunit

*Corresponding author (email: mmaki@agr.nagoya-u.ac.jp)
$(80 \mathrm{kD})$ and the small regulatory subunit $(30 \mathrm{kD})$ revealed the presence of four serially repetitive EF-hands (helix-loop-helix $\mathrm{Ca}^{2+}$-binding motifs) in the $\mathrm{C}$-terminal region of calpain $[1,2]$, and the $\mathrm{Ca}^{2+}$-binding domain was called calmodulin domain for more than a decade.

In 1997, Cygler's group and Narayana's group including Maki independently determined the 3D structures of the rat and pig calpain small subunits by X-ray crystallography $[3,4]$. Surprisingly, the small subunits were found to possess five, not four, EF-hand structures, and the C-terminal fifth EF-hand (EF5) paired up with one from another molecule to 
form a dimer as if EF-hands embrace [5]. In a canonical EF-hand, the calcium ion has a pentagonal bipyramidal coordination (five ligands on the $y z$ plane and two ligands in the $x$ axis), and canonical metal coordination positions are occupied by seven liganding oxygen atoms from the side chain $(x, y, z$, and bidentate $-z)$, peptide carbonyl $(-y)$ and water $(-x)$ in the $\mathrm{Ca}^{2+}$-binding loop (see [6,7] for reviews). However, the newly assigned EF-hand 1 (EF1) lacks one residue in the loop and has a few amino acid substitutions. At the first $\mathrm{Ca}^{2+}$-coordinating position in the loop $(x), \mathrm{EF} 1$ binds $\mathrm{Ca}^{2+}$ by coordinating with the carbonyl oxygen of the peptide bond of Ala in place of the side-chain oxygen of the canonical Asp. The C-terminal region of the calpain large subunit has similar structures, and each EF5 of the large and small subunits pairs to form a calpain heterodimer [8,9]. We proposed the name "penta-EF-hand (PEF)" to indicate a domain found in a group of proteins that possess serially repetitive five EF-hand sequences [10].

\subsection{Finding of apoptosis-linked gene 2 (ALG-2) as a PEF protein}

ALG-2 was one of the six apoptosis-linked genes whose cDNA clones were isolated by the "death-trap" method in which a cDNA library constructed in a mammalian expression vector was used to transfect mouse $\mathrm{T}$ cell hybridoma $3 \mathrm{DO}$ cells in order to protect them from death induced by $\mathrm{T}$ cell receptor stimulation [11]. The obtained ALG-2 cDNA clone turned out to be an anti-sense transcript, and its protective effects were found to be due to depletion of ALG-2, indicating, in other words, that ALG-2 functions as a pro-apoptotic factor [12]. Vito et al. [11] reported that the ALG-2 molecule possesses two canonical EF-hand sequences and demonstrated its $\mathrm{Ca}^{2+}$ - binding capacity by a ${ }^{45} \mathrm{Ca}^{2+}$-ligand blotting (overlay) experiment. A part of the isolated full-length ALG-2 cDNA was identical to a previously reported partial cDNA clone named pMP41 encoding a putative $\mathrm{Ca}^{2+}$-binding protein [13]. We noticed that ALG-2 also contains five EF-hand motifs based on comparison with the amino acid sequences of the C-terminal regions of calpains [10].

\subsection{Evolution of PEF family}

In addition to calpains and ALG-2, sorcin [14,15], grancalcin [16] and peflin [17,18] belong to the PEF family in mammals [19]. Based on the differences in primary structures of the EF1 $\mathrm{Ca}^{2+}$-binding loops, mammalian PEF proteins are classified into two groups: Group I (ALG-2 and peflin) and Group II (calpain subfamily members, sorcin and grancalcin). Sorcin is more similar to grancalcin than to calpain subfamily members. EF1s of Group II proteins commonly have a substitution at the $\mathrm{Ca}^{2+}$-coordinating position $x$ from Asp to Ala and lack one residue in the $\mathrm{Ca}^{2+}$-binding loop.
A closer evolutional relation between sorcin/grancalcin and the calpain subfamily than between sorcin/grancalcin and ALG-2 is supported by the fact that positions of introns are well-conserved among sorcin, grancalcin and calpains but that conservation is limited when intron positions are compared between ALG-2 and calpains [19]. Interestingly, however, phylogenetic analyses based on primary structures have indicated a slightly closer relation of the ALG-2/peflin subfamily to the sorcin/grancalcin subfamily than to the calpain subfamily among all human PEF proteins. This causes erroneous annotation of some PEF proteins in protein sequence databases such as UnitProtKB (http://www. uniprot.org/help/uniprotkb). The sequence of Schistosoma japonicum sorcin (UniProtKB accession code: Q94743) actually corresponds to ALG-2 as evidenced by a high conservation of the EF1 sequence. The sequence of Zea mays grancalcin (UniProtKB accession code: B6SNX9) is more similar to the sequence of Group I PEF proteins in the EF1 sequence, indicating that naming this protein grancalcin is not appropriate. Homologs of PEF I genes have been found in lower animals, plants, fungi and protists. Neither sorcin nor PEF-containing calpains have been found in fungi, suggesting divergence of the ALG-2/sorcin/calpain clan in the animal kingdom [19]. In other words, ancestral ALG-2 is a prototype of PEF proteins in eukaryotes.

Although the lengths of N-terminal tails of ALG-2 proteins are variable (human, 23 aa; chicken, 10 aa; zebra fish, 17 aa; fruit fly, 8 aa), ALG-2 is relatively conserved in animals $(69 \%$ and $89 \%$ identities between humans and fruit fly and between humans and zebra fish, respectively, in the PEF domain of human ALG-2, residues No. 24-191). There are two amino acid substitutions between human and mouse ALG-2 (residue No. 12: Ala in humans vs. Gly in mouse; residue No. 46: Thr in humans vs. Asn in mouse).

\section{Structural analyses of ALG-2}

\subsection{Biochemical properties}

Taking advantage of a $\mathrm{Ca}^{2+}$-dependent aggregation-prone property of ALG-2 at a high protein concentration, recombinant untagged ALG-2 protein $(22 \mathrm{kD})$ can be successfully produced in Escherichia coli and can be easily purified by precipitation with addition of $\mathrm{Ca}^{2+}$ to cell lysates and extraction of the pellets with a calcium chelator [20]. The partially purified protein is further purified by ion exchange chromatography followed by gel filtration. In contrast to sorcin and grancalcin, ALG-2 does not form a stable dimer. $\mathrm{Ca}^{2+}$-free ALG-2 is eluted at the positions of a monomer or slightly larger molecule in the gel filtration [20,21]. However, results of chemical cross-linking experiments in vitro [21] and in vivo [22] suggest the presence of ALG-2 as a dimer. Analytical ultracentrifugation analysis suggests that ALG-2 exists predominantly in a dimer form at higher protein concentrations $\left(5-20 \mu \mathrm{mol} \mathrm{L}^{-1}\right)$ but in equilibrium be- 
tween a monomer and a dimer at protein concentrations less than $1 \mu \mathrm{mol} \mathrm{L}{ }^{-1}$ [22].

Dissociation constants of $\mathrm{Ca}^{2+}$ at two high-affinity binding sites $\left(K_{\mathrm{D}} \sim 2 \mu \mathrm{mol} \mathrm{L}{ }^{-1}\right)$ and one low-affinity binding site $\left(K_{\mathrm{D}} \sim 300 \mu \mathrm{mol} \mathrm{\textrm {L } ^ { - 1 } )}\right.$ were estimated by the flow dialysis method in the presence of $0.5 \%$ Tween 20 [22,23]. When compared with the canonical EF-hand motifs, EF1 and EF3 have sequences that are more conserved than those of EF2, EF4 and EF5 [20]. Analyses of amino acid-substituted mutants of $\mathrm{Ca}^{2+}$-coordinating residues at $-z$ positions of $\mathrm{EF} 1$ (E47A or E47D), EF3 (E114A or E114D) and EF1/EF3 $(\mathrm{E} 47 \mathrm{~A} / \mathrm{E} 114 \mathrm{~A})$ revealed strong $\mathrm{Ca}^{2+}$-binding sites located at EF1 and EF3 [21,22].

Fluorometric analyses using either a fluorescent hydrophobic probe, 2-(p-toluidino)naphthalene-6-sulfonate (TNS), or intrinsic fluorescence of tryptophan revealed that binding to $\mathrm{Ca}^{2+}$ induces conformational changes and exposure of hydrophobic surfaces at a micromolar $\mathrm{Ca}^{2+}$ concentration [20-22]. These biochemical properties agree with those of $\mathrm{Ca}^{2+}$-sensing EF-hand proteins such as calmodulin [24,25].

\subsection{X-ray crystal structures}

Crystals of recombinant ALG-2 proteins were prepared by three independent groups [26-29], and 3D structures were resolved successfully from some of the crystals. The solved structures have the following features common to all PEF proteins whose structures have been solved. (i) Eight $\alpha$-helices instead of ten $\alpha$-helices are present as schematically illustrated for human ALG-2 in Figure 1. Helices $\alpha 4$ and $\alpha 7$ are shared by neighboring EF-hands ( $\alpha 4, \mathrm{EF} 2$ and EF3; $\alpha 7$,

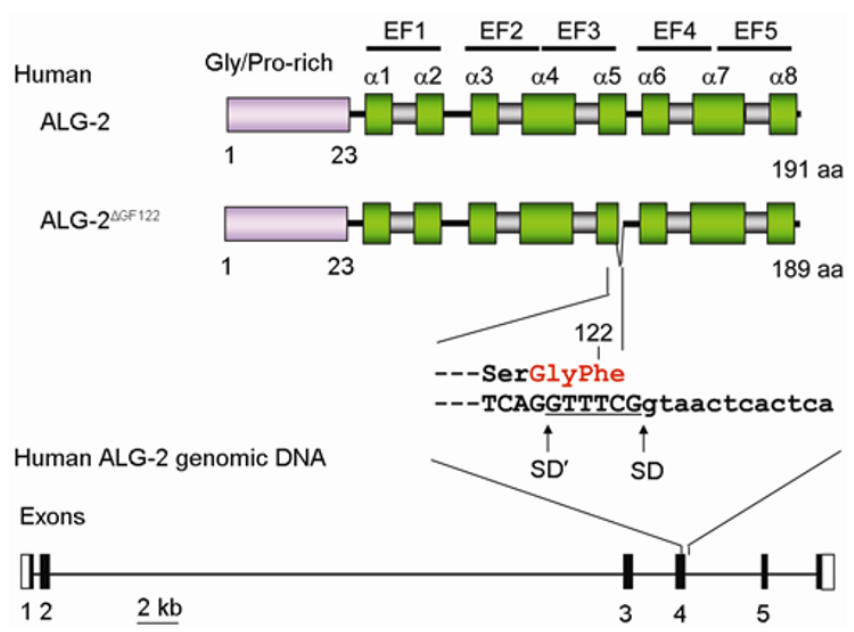

Figure 1 Schematic structures of human ALG-2 proteins and genomic DNA. Alternative splicing at the donor site of Exon 4 generates a six-nucleotide (underlined) shorter mRNA encoding the two-amino acid $\left(\mathrm{Gly}^{121} \mathrm{Phe}^{122}\right.$ )-deleted isoform of ALG-2 (designated ALG-2 ${ }^{\Delta \mathrm{GF} 122}$ ). Five EF-hands (EF1-EF5) are made up of eight $\alpha$-helices $(\alpha 1-\alpha 8)$. SD, splicing donor site; SD', alternative SD. Open boxes and closed boxes in the schematic genomic DNA structure indicate exons encoding untranslated and translated regions, respectively. Nucleotides shown in upper and lower cases indicate exon and intron sequences, respectively.
EF4 and EF5). (ii) Dimerization occurs by pairing of each isolated EF5. An anti-parallel $\beta$-sheet structure is formed between the two EF5s. (iii) Although the overall structure of the region ranging from $\mathrm{EF} 1$ to $\mathrm{EF} 4$ resembles the structure of calmodulin, it is more compact due to the shortness of the central helix connecting EF2 and EF3 ( $\alpha 4)$ in seven residues (corresponding to two turns in the helix) (Figure 2).

Jia et al. [26] crystallized the $\mathrm{Ca}^{2+}$-bound form of mouse des1-20ALG-2 with a buffer containing polyethylene glycol (PEG) 8000 after treatment of full-length recombinant ALG-2 with elastase. They found a continuous electron density in a hydrophobic groove in the solved ALG-2 structure and interpreted it as a Gly/Pro-rich decapeptide derived from the cleaved $\mathrm{N}$-terminus. We also crystallized the $\mathrm{Ca}^{2+}$-bound form of human ALG-2 in the presence of PEG8000 but with genetically engineered N-terminally truncated des3-20ALG-2. Surprisingly, we also observed an electron density in a similar hydrophobic groove [28]. The $\mathrm{Ca}^{2+}$-bound form of des3-23ALG-2 crystallized under different conditions in the absence of PEG8000 showed no such electron density there [29], and it is concluded that a part of the PEG8000 molecule occupied the hydrophobic groove.

In contrast to Group II PEF proteins such as calpain, ALG-2 is unique in that its EF5 binds $\mathrm{Ca}^{2+}$. Lack of coordination by bidentate carboxyl oxygen atoms at the $-z$ position, however, suggests that EF5 has only a weak $\mathrm{Ca}^{2+}$-binding capacity and that $\mathrm{Ca}^{2+}$-binding at this site is non-physiological in the cell. Differences between the 3D structures of the $\mathrm{Ca}^{2+}$-free form and $\mathrm{Ca}^{2+}$-bound form of ALG-2 are small, though an anti-parallel $\beta$-sheet structure is absent in the $\mathrm{Ca}^{2+}$-free form of ALG-2 [28]. Zinc ions bind to EF1 and $\mathrm{EF} 3$ at a coordination similar to that of calcium ions.

\subsection{Comparison of ALG-2 and other PEF proteins}

When the dimeric structure of ALG-2 is compared with the

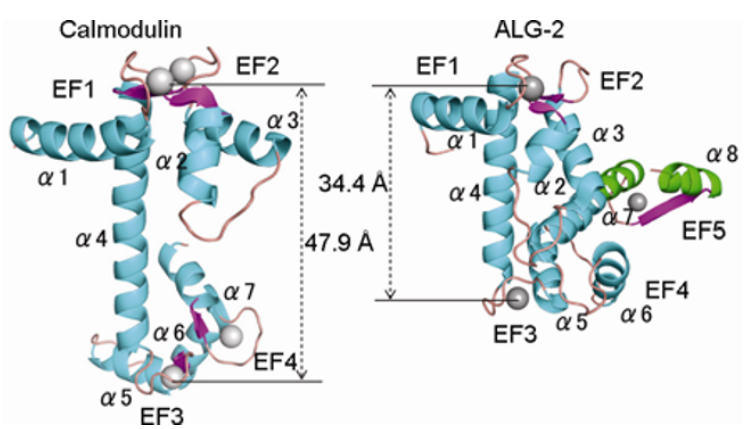

Figure 2 Comparison of the structures of calmodulin and ALG-2 monomer. Figures of 3D structures shown in ribbon representation were generated with PyMol using data deposited in the Protein Data Bank (PDB codes: calmodulin, 1cll; ALG-2, 2zn9). Helices from EF1 to EF4 (in both calmodulin and ALG-2) and those in EF5 (only in ALG-2) are shown in cyan and in green, respectively. Loops and $\beta$-sheet structures are colored in pale pink and magenta, respectively. EF-hand coordinated calcium atoms are shown as gray spheres. Distances between two calcium atoms in EF1 and EF3 are indicated. 
dimeric structures of Group II PEF proteins (grancalcin, sorcin and calpain small subunit), two differences become apparent in overall structures as shown in Figure 3: (i) When anti-parallel $\beta$-sheet-containing loops of EF5s (red) are placed horizontally and $3 \mathrm{D}$ structures are projected onto the paper surface $(X-Y$ plane, top view), the relative positions of EF1s (blue) to the anti-parallel loops are rotated clockwise more in ALG-2, and (ii) the ALG-2 dimer has a larger crevice and smaller interacting surface (side view), possibly being the reason for the aforementioned weaker dimer formation ability than that of Group II PEF proteins.

\subsection{Isoforms}

An isoform of ALG-2 was first reported in the mouse [23]. The isolated cDNA clone designated ALG-2,1 was shorter in six nucleotides corresponding to the two amino acids Gly ${ }^{121} \mathrm{Phe}^{122}$ in comparison with the full-length cDNA clone ALG-2,5. Both transcripts were present in mouse tissues at an approximate ratio of 2:1 (ALG-2,5 vs. ALG-2,1). The same type of isoform lacking 121-GF-122 (designated as ALG-2 $2^{\Delta \mathrm{GF} 122}$ in this article; non-deleted ALG-2 being regarded as wild type for convenience) is also registered in human DNA databases, such as GenBank under accession No. BC110291.1. Since Gly ${ }^{121} \mathrm{Phe}^{122}$ is immediately followed by $\mathrm{Gly}^{123}$ in the amino acid sequence, the deletion of Phe ${ }^{122}$ Gly $^{123}$ is assigned in the results of BLAST searches using the blastp or tblastn program. In the nucleotide sequence of cDNAs, however, the deletion occurs in GGTTTC $\left(\right.$ Gly $^{121} \mathrm{Phe}^{122}$ ) but not in TTCGGC (Phe ${ }^{122} \mathrm{Gly}^{123}$ )

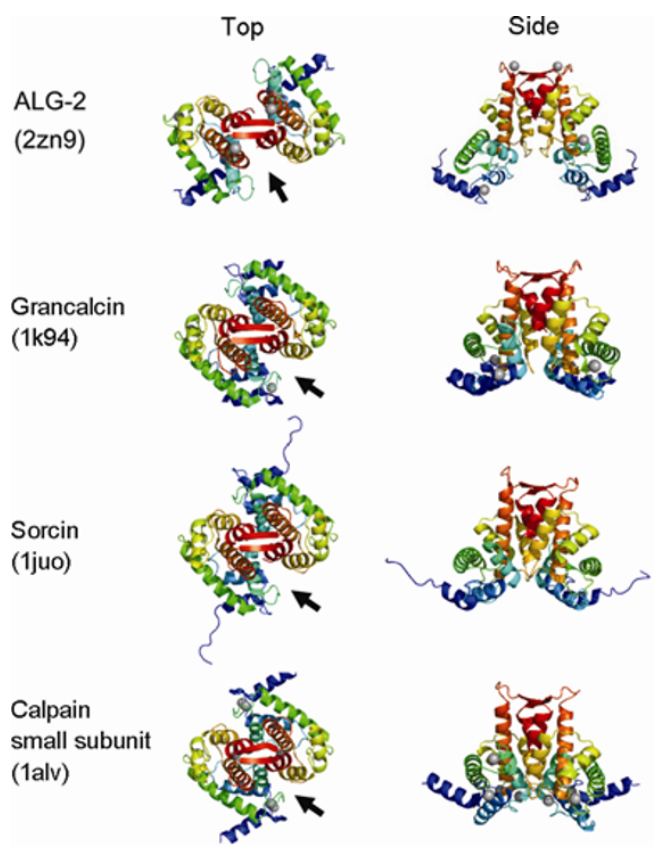

Figure 3 Structural comparison among mammalian PEF proteins. Dimeric structures of PEF domains are shown in ribbon representation. Symmetric two-fold axes are perpendicular to the paper surface in top view that shows anti-parallel $\beta$-sheets of EF5s in red. Arrows indicate orientation of the side view. PDB codes are indicated in parentheses. in the sequence of TCAGGTTTCGGCTAC (corresponding to 120-SGFGY-124).

In the current human EST database, the ratio of retrieved human ALG-2 cDNAs containing nucleotides corresponding to 121-GF-122 and those lacking the corresponding hexanucleotides is approximately $3: 1$. Analysis of the human genome database revealed that the $A L G-2$ gene (symbol, PDCD6; location, 5pter-p15.2) has six exons and that the boundary sequence of Exon 4 and Intron 4 contains an alternative splicing donor site (TCAGGTTTCGgtaactcactca: lower case, intron sequence; underlined sequence, missing by alternative splicing) (Figure 1). The shorter isoform has a lower $\mathrm{Ca}^{2+}$-sensitivity [23].

\section{Interacting proteins}

\subsection{Peflin}

Peflin, a 30-kD PEF protein with a long N-terminal hydrophobic domain, was found as the closest paralog of human ALG-2 [17]. The N-terminal tail has nine repeats of nonapeptide (A/PPGGPYGGP). Orthologs are found in vertebrates and the fruit fly Drosophila but not in the nematode Caenorhabditis elegans. While ALG-2 exists both as a heterodimer with peflin and as a homodimer in the cell, peflin exists only as a heterodimer with ALG-2 [18]. The heterodimerization occurs through their EF5s [30]. The heterodimer formed between peflin and ALG-2 dissociates in the presence of $\mathrm{Ca}^{2+}$ [18], probably due to binding of $\mathrm{Ca}^{2+}$ to EF5 of ALG-2 at a high $\mathrm{Ca}^{2+}$ concentration. Peflin/ALG-2 heterodimer may be stable under physiological conditions. However, soluble recombinant proteins of peflin are not yet available, and knowledge of biochemical properties and functions is limited.

\section{2 $\mathrm{Ca}^{2+}$-dependent interaction}

ALG-2 functions as a $\mathrm{Ca}^{2+}$ sensor by changing its conformation in a $\mathrm{Ca}^{2+}$-dependent manner at the micromolar level of $\mathrm{Ca}^{2+}$ as described above. This conformational change enables ALG-2 to interact with various proteins. ALIX (ALG-2-interacing protein $\mathrm{X}$, also named AIP1) was the first protein that was shown to interact with ALG-2 in a $\mathrm{Ca}^{2+}$-dependent fashion [31,32]. The $\mathrm{C}$-terminal region containing a Pro-rich sequence was shown to be essential for the binding. Subsequently, annexin A11 (previously designated as annexin XI) and annexin A7 (annexin VII) were shown to interact with ALG-2 [33,34]. The N-terminal regions of these annexins also have Pro-rich sequences. A number of ALG-2-interacting proteins have been screened by the yeast two-hybrid method and mass-spectrometric analyses of products of co-immunoprecipitation or pulldown with glutathione-S-transferase (GST)-fused ALG-2. Combinations of different methods are necessary to further validate the interactions of candidate proteins with ALG-2 
in vitro and/or in vivo. Thus, when searching current protein-protein interaction databases such as http://string-db. org/ and http://www.ebi.ac.uk/intact, results should be carefully interpreted.

A report of interaction of "ALG-2" with Fas/CD95 (one of the death receptors) by Jung et al. [35] was sensational not only from the viewpoint of potential roles of ALG-2 in apoptosis through Fas signaling but also in that there were changes in the cellular distribution of "ALG-2" from the plasma membrane to cytosol upon Fas stimulation with a concomitant limited cleavage of "ALG-2". However, Fas-ALG-2 interaction was later denied by the group of Berchtold who clearly demonstrated that the commercially available "anti-ALG-2 monoclonal antibody" used by Jung et al. [35] did not react with ALG-2 but erroneously reacted with the co-chaperone protein p23 [36,37]. Ask1 [38] and Raf-1 [39], protein kinases involved in apoptosis and in cell signaling, and mucolipin-1 [40], an ion channel involved in mucolipidosis, were shown to physically associate with ALG-2, but these proteins contain no PRRs. While the binding sites were not investigated in the case of Ask1 and Raf-1, a region containing 37-EEEDLRRRLKYFF-49 in mucolipin-1 was essential for interaction, but the minimal binding region requires a neighboring region on either the $\mathrm{N}$-terminal or C-terminal side.

\subsection{ALG-2-binding motifs}

An ALG-2-binding site, which was determined by analyses of serially deleted and substituted mutants, contains four tandem repeats of $\mathrm{P} X \mathrm{Y}(X$, variable amino acid residue) in the PRR of ALIX [41]. By in silico searching for a similar $\mathrm{PXY}$ sequence in the protein sequence databases, phospholipid scramblase 3 (PLSCR3) became a candidate of a novel ALG-2-interacting protein [42]. Unexpectedly, mutational analyses revealed that PLSCR3 has two ALG-2-binding sites (ABS-1 and ABS-2): one (ABS-1) similar to the ALG-2-binding site in ALIX and the other (ABS-2) different from that of ALIX. ABS-2 was later found to be similar to the ALG-2-binding site of Sec31A [43]. While ALIX and ABS-1 of PLSCR3 do not bind the alternatively spliced shorter isoform ALG-2 ${ }^{\triangle \mathrm{GF1} 122}[23,42]$, both Sec31A and ABS-2 of PLSCR 3 can bind not only ALG-2 but also ALG-2 ${ }^{\Delta \mathrm{GF} 122}[42,44]$. An ABS-2-oligopeptide-immobilized column was proven to be useful for $\mathrm{Ca}^{2+}$-dependent affinity purification of recombinant ALG-2 proteins including ALG-2 ${ }^{\triangle \mathrm{GF} 122}$ [42]. Comparison of the identified binding sequences among ALIX, PLSCR3 and Sec31A revealed two consensus core ALG-2-binding motifs (ABM-1, PPYPXXPGYP; ABM-2, PXPGF) (Figure 4). Although the exact binding sites are not yet known, several other ALG-2binding proteins also contain sequences rich in Pro, Gly and Tyr, such as annexin A7 (4-PGYPPTGYPP-15), annexin A11 (4-PGYPPPPGGYPP-15), TSG101 (188-CPYPPGGPYPA198) [45], and Scotin (190-PYPMQYPPPYP-200) [46]

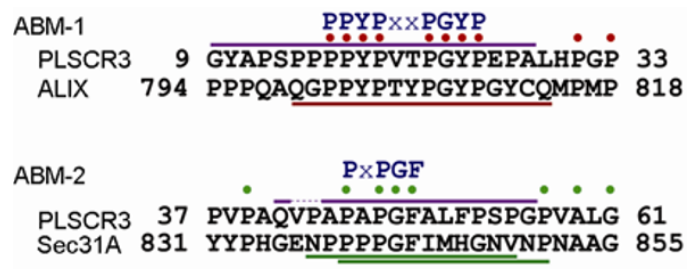

Figure 4 Alignment of ALG-2-binding sites in PLSCR3, ALIX and Sec31A. Minimal amino sequences sufficient for interaction with ALG-2 in in vitro binding assays using either synthetic oligopeptides (PLSCR3, ALIX) or glutathione-S-transferase (GST)-fused peptides (Sec31A) are shown by bars above and below the sequences (purple, PLSCR3; brown, ALIX; green, Sec31A). Identical amino acid residues between each pair are indicated by closed circles, above which consensus sequences for minimal binding (ALG-2-binding motif, ABM) are shown.

suggesting a consensus sequence of $\operatorname{PPYP}(X) n Y \mathrm{P}(X$, variable amino acids, $n=3-6)$. Optimum sequence and number of residues between two YPs may depend on surrounding residues. On the other hand, RBM22 [47] has an ABM-2-like sequence 373-PPPPPGFGPHMFHPMGPPPP-392, whose binding ability remains to be clarified. The effects of amino acid substitutions of ALG-2 on ability of binding to target proteins are significantly different not only between ABM-1 type proteins (ALIX, annexin A7, annexin A11 and TSG101) and ABM-2 type protein (Sec31A) but also slightly different even among ABM-1 type proteins $[42,48]$. Thus, the mode of binding appears to be flexible.

\section{4 $\mathrm{Ca}^{2+} / \mathrm{EF3-driven} \mathrm{arginine} \mathrm{switch} \mathrm{mechanism}$}

The structure of the complex between ALG-2 and an ALIX oligopeptide was successfully solved at $2.2 \AA$ resolution by crystallization of N-terminally truncated ALG-2 (des323ALG-2) and a 16-residue ALIX oligopeptide (1QGPPYPTYPGYPGYSQ-16, cysteine substituted with serine) corresponding to ALIX 799-814 in the presence of $\mathrm{Zn}^{2+}$ instead of $\mathrm{Ca}^{2+}$ (PDB code: 2zne) $[28,49]$. The ALIX peptide is seen in a groove that contains two peptide-binding hydrophobic pockets (Pockets 1 and 2) in ALG-2 (Figure 5). Pocket 1 is formed by residues from the $\alpha 5-\alpha 6$ loop and those from EF5 ( $\alpha 7$ to $\alpha 8$ ) (Figure 6). Y180 from the other ALG-2 molecule in the dimer also constitutes the bottom of Pocket 1 . Pocket 2 is formed by residues from $\alpha 3$ to $\alpha 5$ including F122 and from $\alpha 7$. Polyproline II (PPII) left-handed helices are often observed in Pro-rich regions [50], but the solved ALIX peptide structure does not form such a PPII helix. In vitro binding assays using mutant ALIX peptides revealed that 3-PPYP-6, binding to Pocket 1 , is the most critical segment. Pocket 2 accepts 11-YPG-13 and Q16. Among the three YPs, the central 8-YP-9 does not bind to the hydrophobic pockets but loops out from the surface of Pocket 2, supporting the consensus sequence for ABM-1, $\operatorname{PPYP}(X) n Y \mathrm{Y}$, where the number of residues between two YPs is variable ( $n=4$, ALIX and PLSCR3).

The $\mathrm{Ca}^{2+}$ (also $\mathrm{Zn}^{2+}$ )-dependent binding of ALG-2 to the 


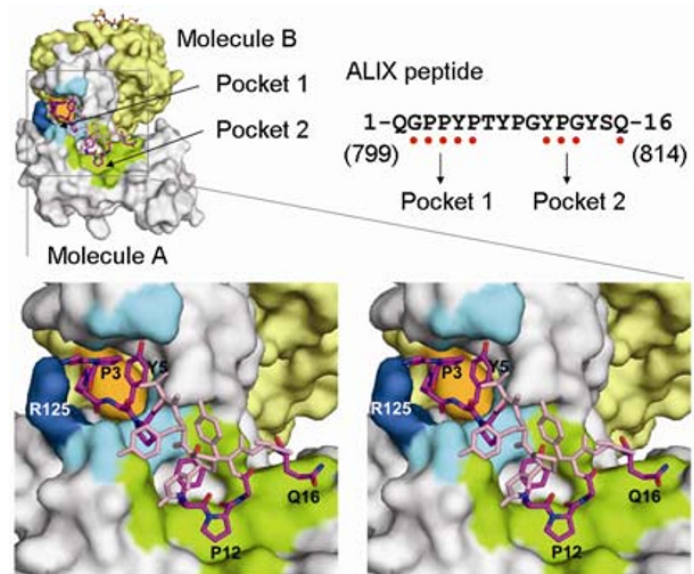

Figure 5 3D structure of the complex between ALG-2 and ALIX peptide. Two ALIX peptides (stick representation) bind to the ALG-2 dimer (surface representation: Molecule A, largely gray and colored in cyan for Pocket 1 and light green for Pocket 2; Molecule B, light yellow; PDB code, 2zne). Pocket 1 door, R125 in Molecule A, and Y180(B), coming from Molecule B and creating a hydrophobic bottom of Pocket 1, are colored in blue and orange, respectively. The ALIX peptide bound to Molecule A is shown in stick representation in two different colors for carbon atoms: magenta, ALG-2-interacting residues; pale pink, non-interacting residues. Contact area is enlarged and shown in parallel stereoview. Nitrogen atoms and oxygen atoms are also colored in blue and red, respectively, for the ALG-2-interacting residues, but they are in the same color (pale pink) as carbon atoms in the non-interacting residues.

ALIX peptide is explained as shown in Figure 6: (i) Binding of $\mathrm{Ca}^{2+}$ (or $\mathrm{Zn}^{2+}$ ) to EF3 causes change in the side chain configuration of R125 that is located at the loop connecting $\alpha 5$ and $\alpha 6$; (ii) opening of Pocket 1 , (iii) entry of the peptide, and (iv) re-closure of Pocket 1 by the R125 side chain and completion of the peptide trapping. Thus, R125 is a key element of the open-close mechanism of Pocket 1 and acts as a switch driven by $\mathrm{Ca}^{2+} / \mathrm{EF} 3$ conformational change. In the crystal structure of ALG-2 $2^{\Delta \mathrm{GF} 122}$ in the $\mathrm{Ca}^{2+}$-bound form [48], deletion of the two amino acid residues, 121-GF-122, truncates $\alpha 5$ and 120-SGY-122 forms either 310 -helix (molecule A) or turn and bend (molecule B), affecting configuration of the R123 side chain (corresponding to R125 in ALG-2 ${ }^{\mathrm{WT}}$ ). A hydrogen bond between a carbonyl oxygen atom of S120 $\left(\mathrm{O}^{\mathrm{S} 120}\right)$ and guanidino nitrogen atom of R125 $\left(\mathrm{NH} 2^{\mathrm{R} 125}\right)$ is observed in the $\mathrm{Ca}^{2+}$-bound form but not in the $\mathrm{Ca}^{2+}$-free form of ALG-2 ${ }^{\mathrm{WT}}$ [28]. A hydrogen bond between $\mathrm{O}^{\mathrm{S} 120}$ and $\mathrm{NH}_{2}{ }^{\mathrm{R} 123}$ is not formed in the $\mathrm{Ca}^{2+}$-bound form of ALG-2 $2^{\triangle \mathrm{GF} 122}$ either [48]. While "R125 door" is opened by $\mathrm{Ca}^{2+}$, "R123 door" of the isoform is kept closed. Substitution of F122 with alanine does not affect the configuration of R125, and the "R125" door is opened in the metal-bound form (Figure 6). Indeed, the mutant ALG$2^{\mathrm{F} 122 \mathrm{~A}}$ exhibited a stronger ALIX-binding activity. Removal of the bulky side chain of F122 created an additional open space in Pocket 2 and abolished inter-helix interactions with W95 and V98 (present in $\alpha 4$ ), and $\alpha 5$ inclined away from $\alpha 4$ to expand Pocket 2, suggesting acquirement of more appropriate positioning of the interacting residues to accept the target protein [48]. The structural basis for the $\mathrm{Ca}^{2+}$-dependent binding of ALG-2 to ABM-2 peptides remains to be established. Since ALG-2 $2^{\triangle \mathrm{GF} 122}$ and Y180A mutant, incapable of dimerization, maintain the ability for binding to Sec31A and PLSCR3, ABM-2 peptides appear to bind to a hydrophobic pocket(s) different from at least Pocket 1.

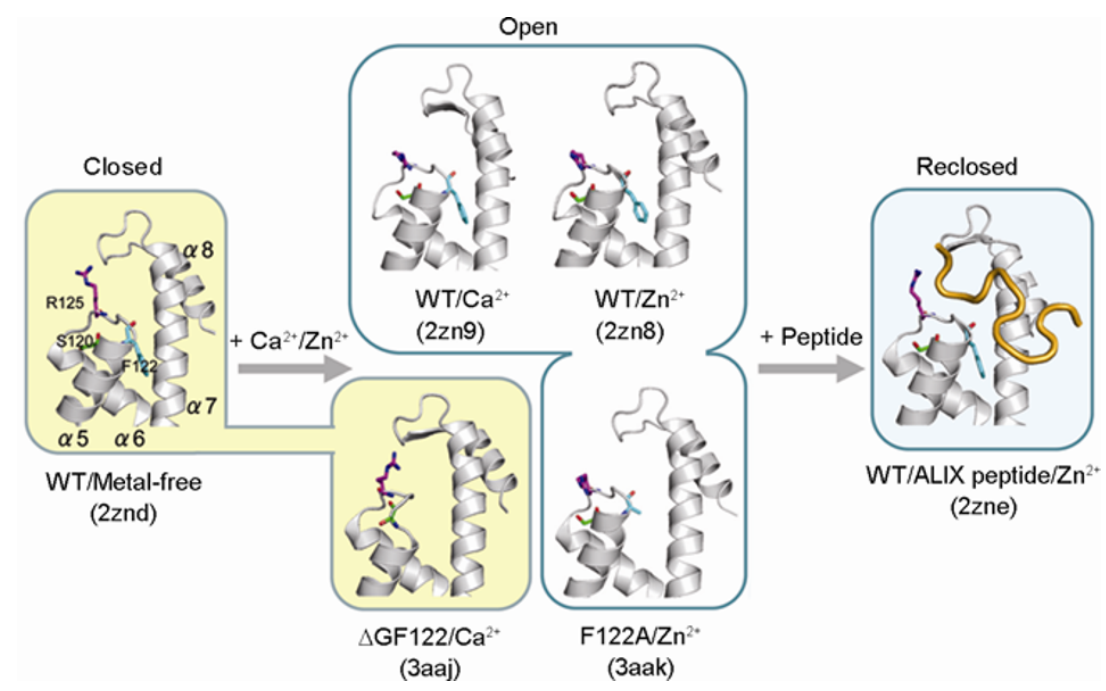

Figure 6 Model of $\mathrm{Ca}^{2+} / \mathrm{EF} 3$-driven arginine switch mechanism. In the metal free form of wild-type ALG-2 (designated WT), the flexible side chain of R125, located in the loop connecting helices $\alpha 5$ and $\alpha 6$, blocks the mouth of the primary hydrophobic pocket (Pocket 1 ) and interferes with entry of the target peptide. Binding of $\mathrm{Ca}^{2+}$ or $\mathrm{Zn}^{2+}$ to EF3 induces a small local movement of helix $\alpha 5$ and relays configuration change of the side chain of R125 forming a hydrogen bond with the carbonyl oxygen atom of S120, resulting in opening of Pocket 1 and accepting the ALIX peptide. While the side chain of R123 in the $\mathrm{Ca}^{2+}$-bound form of ALG-2 ${ }^{\Delta \mathrm{GF} 122}$ (corresponding to R125 of ALG-2) keeps blocking the pocket, Pocket 1 is opened in the $\mathrm{Zn}^{2+}$-bound form of the amino acid substitution mutant F122A. PDB codes are indicated in parentheses. Gray fancy ribbons represent $\alpha$-helices and $\beta$-sheets. ALIX peptide is shown by a light orange cord. 


\section{$4 \mathrm{Ca}^{2+}$-dependent adaptor}

\subsection{Subcellular localization of ALG-2}

Fluorescence microscopic analyses of ALG-2 using specific antibodies have revealed that ALG-2 localizes in both the cytoplasm and nucleus in the cell $[18,41]$. The presence of ALG-2 in the nucleus implies certain functions in gene expression regulation, precursor RNA processing or nuclear export of RNAs and/or proteins. RBM22, a nuclear protein that contains RNA-binding motif, may work together with ALG-2 [47]. It remains unknown whether RBM22 is a major target of ALG-2 in the nucleus. Although ALG-2 does not bind to liposomes by itself in the presence of $\mathrm{Ca}^{2+}$ in vitro, addition of $\mathrm{Ca}^{2+}$ to cell lysates causes translocation of ALG-2 to membranous fractions, suggesting binding of ALG-2 to membrane-localizing proteins, which reside on the membrane either as integral membrane proteins or as peripheral proteins recruited to membranes upon stimulation. Since each ALG-2 monomer molecule in the dimer accepts one ALIX peptide molecule and the dimer accepts two peptides (Figure 5), this stoichiometry raises the possibility that an ALG-2 dimer links two binding molecules and functions as a $\mathrm{Ca}^{2+}$-dependent adaptor protein on the membranes or in their vicinity. This adaptor function of ALG-2 may be effective for stabilizing two weakly interacting proteins or for bridging two unrelated proteins localizing together in the vicinity of the membrane or scaffolds (Figure 7).

\subsection{Bridging ESCRT-I and ESCRT-III by the ALIX/ ALG-2 complex}

Plasma membrane proteins are endocytosed and delivered to endosomes, from which they are recycled back to the plasma membrane or further transported to lysosomes for degradation. Ubiquitinated cargo proteins are sorted on the complex required for transport) system, and multivesicular
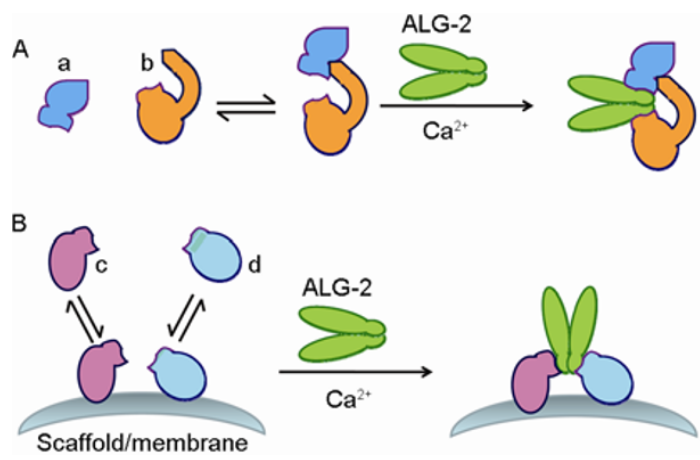

Figure $7 \mathrm{Ca}^{2+}$-dependent adaptor function of ALG-2. Models of ALG-2 functioning as a $\mathrm{Ca}^{2+}$-dependent adaptor are illustrated in two cases. A, Two ALG-2-binding proteins $(\mathrm{a}, \mathrm{b})$ weakly associate with each other, and the complex is stabilized by ALG-2 in the presence of $\mathrm{Ca}^{2+}$. $\mathrm{B}$, Two ALG-2-binding proteins ( $\mathrm{c}$ and d) do not associate by themselves but they are bridged by ALG- 2 on scaffold proteins or membranes that they associate with directly or indirectly. bodies (MVBs) are formed by the invagination and scission of buds from the limiting membrane of the endosome into the lumen [51]. Among the four ESCRT complexes, cargoes are sequentially recognized by ESCRT-0, -I, and -II, each of which contains a different ubiquitin-binding subunit. ESCRT-III cleaves the bud necks to form intraluminal vesicles [52]. The ESCRT system also functions in release of enveloped RNA virus particles from the plasma membrane and scission of two daughter cells at cytokinesis $[53,54]$.

ALIX is an accessory protein of the ESCRT system [52]. While an N-terminally located Brol domain of ALIX associates with CHMP4 (human ortholog of yeast Snf7, a major component of ESCRT-III) [55], the PSAP-sequence in the Pro-rich region of ALIX interacts with a UEV domain of TSG101 (a ubiquitin- and PTAP-motif-binding subunit of ESCRT-I), but this ALIX-TSG101 interaction is weak when compared with interaction between the UEV domain and HIV-1 p6, containing a PTAP motif, in in vitro binding assays using a surface plasmon resonance (SPR) biosensor (ALIX peptide 714-723, $K_{\mathrm{D}}=142 \mu \mathrm{mol} \mathrm{L}{ }^{-1}$; unubiquitinated p6 peptide, $K_{\mathrm{D}}=27 \mu \mathrm{mol} \mathrm{L}^{-1}$; ubiquitinated p6 peptide, $K_{\mathrm{D}}=2.3 \mu \mathrm{mol} \mathrm{L}{ }^{-1}$ ) [56,57]. Using lysates of cells exogenously expressing Strep-tagged ALIX, endogenous TSG101 has been shown to be recovered in the pulldown products of Strep-ALIX in the presence of $\mathrm{Ca}^{2+}$ but not in the absence of $\mathrm{Ca}^{2+}$ [58]. Since ALG-2 directly associates with TSG101 in a $\mathrm{Ca}^{2+}$-dependent manner [45], ALG-2 was predicted to be a $\mathrm{Ca}^{2+}$-dependent factor of ALIX-TSG101 interaction. Indeed, we found that the ALG-2-binding site, but not the PSAP sequence, in ALIX is important for $\mathrm{Ca}^{2+}$-dependent ALIX-TSG101 complex formation and that depletion of ALG-2 by RNA interference (RNAi) abolished the interaction [59]. Moreover, exogenous expression of a wild-type ALG-2 construct could restore the $\mathrm{Ca}^{2+}$-dependent ALIXTSG101 interaction in endogenous ALG-2-depleted cells, but constructs of a dimerization-defective mutant (Y180A substitution) and a shorter isoform ALG-2 $2^{\Delta \mathrm{GF} 122}$ could not. We propose that a $\mathrm{Ca}^{2+}$-loaded dimeric form of ALG-2 stabilizes the complex between ALIX and TSG101 and functions as a $\mathrm{Ca}^{2+}$-adaptor protein as in the case of Figure $7 \mathrm{~A}$. ALIX directly associates with TSG101 through a weak interaction between the PSAP sequence in ALIX and the UEV domain of TSG101, and this complex is stabilized by the $\mathrm{Ca}^{2+}$-bound ALG-2 dimer, which binds to the Pro-rich regions of the respective target proteins.

\subsection{Function of ALG-2 in ER-to-Golgi transport system}

By staining with antibodies of cytoplasmic marker proteins, ALG-2 was found to partially colocalized with Sec31A, a component of COPII, in a punctate pattern at endoplasmic reticulum (ER) exit sites [44,60]. Live-cell time-lapse imaging revealed that fluorescent protein-fused ALG-2 is recruited to ER exit sites in accordance with $\mathrm{Ca}^{2+}$ oscillation triggered by a $\mathrm{Ca}^{2+}$-mobilization reagent such as histamine 
[43,61]. Furthermore, fluorescence recovery after photobleaching (FRAP) analysis indicated that deletion of ALG-2-binding site in Sec31A (amino acid residues 839851) reduced the high-affinity (slower dissociation) population of Sec31A at the ER exit sites, suggesting that the capability of ALG-2 binding is one of the key determinants of the retention kinetics of Sec31A at ER exit sites [43]. ALG-2 and calcium ions inhibit COPII vesicle fusions [62]. Recently, we have found that annexin $\mathrm{A} 11$, a $\mathrm{Ca}^{2+} /$ phospholipid-binding protein, is involved in this ER-to-Golgi transport pathway and that ALG-2 bridges Sec31A and annexin A11 at ER exit sites [63].

\section{Physiological functions of ALG-2}

Regardless of the original cloning report of ALG-2 cDNA in a screen for genes involved in cell death [11], ALG-2deficient mice generated by the same group showed no abnormality in development and immune functions [64]. Nevertheless, ALG-2 has been suggested to work in cell death during ER stress [65]. ALIX and ALG-2 appear to work in $\mathrm{K}^{+}$-deprived neuronal cells [66], and they interact directly or indirectly with the $\mathrm{TNF} \alpha$ receptor-1 (TNF-R1) signaling complex [67]. Høj et al. [68] reported that ALG-2 was required for normal cell division because deficiency of ALG-2 by the RNA interference method resulted in G2/M cell cycle phase accumulation and cell death. ALG-2 has been suggested to be involved in cancer [69]. However, a cause-effect relationship is not clear. The gene of ALG-2 (PDCD6, chromosomal locus at 5p15.33) is a potential prognostic marker for malignant progression in pulmonary adenocarcinoma with bronchioloalveolar features, and patients with high expression levels of PDCD6 have low survival rates [70]. By contrast, low expression level of PDCD6 is correlated with poor overall survival of gastric cancer patients [71]. Since malfunction of MVB sorting and ER-to-Golgi transport affects normal cell growth, dysregulation of the delicate balance between proliferation and cell death may lead to malignant progression of tumors.

\section{Conclusion}

EF-hand proteins have similar helix-loop-helix structures, but conformational states with respect to angles and distances between the two helices and their changes in response to $\mathrm{Ca}^{2+}$ binding are very diverse [72]. In the case of calmodulin, each pair of EF1-EF2 (N-lobe) and EF3-EF4 (C-lobe) changes its conformation from "closed" to "open" state upon $\mathrm{Ca}^{2+}$ binding and exhibits a further gross change in relative stereotypic position by bending of the central helix connecting EF2 and EF3 in such a way that the two lobes grab the targeting peptide [73]. On the other hand, ALG-2 does not exhibit a significant change from the closed conformational state in the absence of $\mathrm{Ca}^{2+}$ to the open conformational state in the presence of $\mathrm{Ca}^{2+}$. Nevertheless, binding of $\mathrm{Ca}^{2+}$ or $\mathrm{Zn}^{2+}$ to EF3 causes a small shift of $\alpha 5$ and leads to change in configuration of the R125 side chain to move sufficiently for making a primary hydrophobic pocket accessible to the critical PPYP motif found in ALIX. This novel $\mathrm{Ca}^{2+} / \mathrm{EF} 3$-driven arginine switch mechanism explains how ALG-2 is activated by $\mathrm{Ca}^{2+}$ to bind to its target proteins of at least ABM-1 type proteins.

There has been a gradual annual increase in the number of published articles on ALG-2-interacting proteins. Like calmodulin, ALG-2 may interact with various proteins containing flexible recognition motifs with different affinities. Data in the genome-wide protein-protein-network (interactome) database obtained by yeast two-hybrid and mass spectrometric analyses of affinity-captured proteins are helpful for inferring physiological functions of ALG-2. However, elimination of false-positive interactions by careful assessment is crucial to avoid misleading to a wrong direction of research. We propose a new concept of ALG-2: a $\mathrm{Ca}^{2+}$-dependent adaptor. Among the interacting proteins, it is necessary to investigate in which combinations ALG-2 works as an adaptor. Co-localization seems one of the key points for evaluating physiologically important partners. In certain cases, ALG-2 may promote oligomerization of interacting proteins. Although detection of interactions of endogenous proteins with antibodies is the best way to validate physical interactions, a scarce protein may not be detectable. It is possible that as-yet-unidentified ALG-2interacting proteins with high affinity and low abundance, rather than already reported proteins reviewed in this article, play critical roles in cell functions. However, weak binding does not necessarily mean that the interaction is unphysiological. Greater rates of dissociation as well as association make it possible to respond more rapidly to the oscillating $\mathrm{Ca}^{2+}$ concentration levels.

We thank members of the Laboratory of Molecular and Cellular Regulation, Nagoya University Graduate School of Bioagricultural Sciences (MCR-NUGSBS) for their contributions to biochemical and molecular cell biological studies. We also thank Tatsutoshi Inuzuka (MCR-NUGSBS) and Drs. Soichi Wakatsuki and Masato Kawasaki (KEK, Tsukuba) for X-ray crystallography.

1 Ohno S, Emori Y, Imajoh S, et al. Evolutionary origin of a calcium-dependent protease by fusion of genes for a thiol protease and a calcium-binding protein? Nature, 1984, 312: 566-570

2 Sakihama T, Kakidani $\mathrm{H}$, Zenita $\mathrm{K}$, et al. A putative $\mathrm{Ca}^{2+}$-binding protein: structure of the light subunit of porcine calpain elucidated by molecular cloning and protein sequence analysis. Proc Natl Acad Sci USA, 1985, 82: 6075-6079

3 Blanchard H, Grochulski P, Li Y, et al. Structure of a calpain $\mathrm{Ca}^{2+}$-binding domain reveals a novel EF-hand and $\mathrm{Ca}^{2+}$-induced conformational changes. Nat Struct Biol, 1997, 4: 532-538

4 Lin G D, Chattopadhyay D, Maki M, et al. Crystal structure of calcium bound domain VI of calpain at $1.9 \AA$ resolution and its role in enzyme assembly, regulation, and inhibitor binding. Nat Struct Biol, 1997, 4: 539-547 
5 Kretsinger R H. EF-hands embrace. Nat Struct Biol, 1997, 4: 514516

6 Kawasaki H, Nakayama S, Kretsinger R H. Classification and evolution of EF-hand proteins. Biometals, 1998, 11: 277-295

7 Grabarek Z. Structural basis for diversity of the EF-hand calciumbinding proteins. J Mol Biol, 2006, 359: 509-525

8 Hosfield C M, Elce J S, Davies P L, et al. Crystal structure of calpain reveals the structural basis for $\mathrm{Ca}^{2+}$-dependent protease activity and a novel mode of enzyme activation. EMBO J, 1999, 18: 6880-6889

9 Strobl S, Fernandez-Catalan C, Braun M, et al. The crystal structure of calcium-free human m-calpain suggests an electrostatic switch mechanism for activation by calcium. Proc Natl Acad Sci USA, 2000, 97: 588-592

10 Maki M, Narayana S V, Hitomi K. A growing family of the $\mathrm{Ca}^{2+}$-binding proteins with five EF-hand motifs. Biochem J, 1997, 328: 718-720

11 Vito $\mathrm{P}$, Lacanà E, D'Adamio L. Interfering with apoptosis: $\mathrm{Ca}^{2+}$ binding protein ALG-2 and Alzheimer's disease gene ALG-3. Science, 1996, 271: 521-525

12 Lacanà $\mathrm{E}$, Ganjei $\mathrm{J} \mathrm{K}$, Vito $\mathrm{P}$, et al. Dissociation of apoptosis and activation of IL-1 $\beta$-converting enzyme/Ced-3 proteases by ALG-2 and the truncated Alzheimer's gene ALG-3. J Immunol, 1997, 158: 51295135

13 Kageyama H, Shimizu M, Tokunaga K, et al. A partial cDNA for a novel protein which has a typical EF-hand structure. Biochim Biophys Acta, 1989, 1008: 255-257

14 Van der Bliek A M, Meyers M B, Biedler J L, et al. A 22-kD protein (sorcin/V19) encoded by an amplified gene in multidrug-resistant cells, is homologous to the calcium-binding light chain of calpain. EMBO J, 1986, 5: 3201-3208

15 Hamada H, Okochi E, Oh-hara T, et al. Purification of the Mr 22000 calcium-binding protein (sorcin) associated with multidrug resistance and its detection with monoclonal antibodies. Cancer Res, 1988, 48: 3173-3178

16 Boyhan A, Casimir C M, French J K, et al. Molecular cloning and characterization of grancalcin, a novel EF-hand calcium-binding protein abundant in neutrophils and monocytes. J Biol Chem, 1992, 267: 2928-2933

17 Kitaura Y, Watanabe M, Satoh H, et al. Peflin, a novel member of the five-EF-hand-protein family, is similar to the apoptosis-linked gene 2 (ALG-2) protein but possesses nonapeptide repeats in the N-terminal hydrophobic region. Biochem Biophys Res Commun, 1999, 263: 68-75

18 Kitaura Y, Matsumoto S, Satoh H, et al. Peflin and ALG-2, members of the penta-EF-hand protein family, form a heterodimer that dissociates in $\mathrm{a} \mathrm{Ca}^{2+}$-dependent manner. J Biol Chem, 2001, 276: 1405314058

19 Maki M, Kitaura Y, Satoh H, et al. Structures, functions and molecular evolution of the penta-EF-hand $\mathrm{Ca}^{2+}$-binding proteins. Biochim Biophys Acta, 2002, 1600: 51-60

20 Maki M, Yamaguchi K, Kitaura Y, et al. Calcium-induced exposure of a hydrophobic surface of mouse ALG-2, which is a member of the penta-EF-hand protein family. J Biochem, 1998, 124: 1170-1177

21 Lo K W, Zhang Q, Li M, et al. Apoptosis-linked gene product ALG-2 is a new member of the calpain small subunit subfamily of $\mathrm{Ca}^{2+}$-binding proteins. Biochemistry, 1999, 38: 7498-7508

22 Subramanian L, Crabb J W, Cox J, et al. $\mathrm{Ca}^{2+}$ binding to EF hands 1 and 3 is essential for the interaction of apoptosis-linked gene-2 with Alix/AIP1 in ocular melanoma. Biochemistry, 2004, 43: 1117511186

23 Tarabykina S, Møller A L, Durussel I, et al. Two forms of the apoptosis-linked protein ALG-2 with different $\mathrm{Ca}^{2+}$ affinities and target recognition. J Biol Chem, 2000, 275: 10514-10518

24 LaPorte D C, Wierman B M, Storm D R. Calcium-induced exposure of a hydrophobic surface on calmodulin. Biochemistry, 1980, 19: 3814-3819

25 Tanaka T, Hidaka H. Hydrophobic regions function in calmodulin-enzyme(s) interactions. J Biol Chem, 1980, 255: 11078-11080

26 Jia J, Tarabykina S, Hansen C, et al. Structure of apoptosis-linked protein ALG-2: insights into $\mathrm{Ca}^{2+}$-induced changes in penta-EF-hand proteins. Structure, 2001b, 9: 267-275

27 Wu F, Zhang M, Gong W. Crystallization and preliminary crystallographic studies of an apoptosis-linked calcium-binding protein ALG-2. Acta Crystallogr D Biol Crystallogr, 2001, 57: 1162-1163

28 Suzuki H, Kawasaki M, Inuzuka T, et al. Structural basis for $\mathrm{Ca}^{2+}$-dependent formation of ALG-2/Alix peptide complex: $\mathrm{Ca}^{2+}$ / EF3-driven arginine switch mechanism. Structure, 2008, 16: 1562-1573

29 Suzuki H, Kawasaki M, Kakiuchi T, et al. Crystallization and X-ray diffraction analysis of N-terminally truncated human ALG-2. Acta Crystallogr Sect F Struct Biol Cryst Commun, 2008, 64: 974-977

30 Kitaura Y, Satoh H, Takahashi H, et al. Both ALG-2 and peflin, penta-EF-hand (PEF) proteins, are stabilized by dimerization through their fifth EF-hand regions. Arch Biochem Biophys, 2002, 399: 12-18

31 Missotten M, Nichols A, Rieger K, et al. Alix, a novel mouse protein undergoing calcium-dependent interaction with the apoptosis-linked-gene 2 (ALG-2) protein. Cell Death Differ, 1999, 6: 124129

32 Vito P, Pellegrini L, Guiet C, et al. Cloning of AIP1, a novel protein that associates with the apoptosis-linked gene ALG-2 in a $\mathrm{Ca}^{2+}$ dependent reaction. J Biol Chem, 1999, 274: 1533-1540

33 Satoh H, Shibata H, Nakano Y, et al. ALG-2 interacts with the amino-terminal domain of annexin $\mathrm{XI}$ in a $\mathrm{Ca}^{2+}$-dependent manner. Biochem Biophys Res Commun, 2002, 291: 1166-1172

34 Satoh H, Nakano Y, Shibata H, et al. The penta-EF-hand domain of ALG-2 interacts with amino-terminal domains of both annexin VII and annexin XI in a $\mathrm{Ca}^{2+}$-dependent manner. Biochim Biophys Acta, 2002, 1600: 61-67

35 Jung Y S, Kim K S, Kim K D, et al.. Apoptosis-linked gene 2 binds to the death domain of Fas and dissociates from Fas during Fas-mediated apoptosis in Jurkat cells. Biochem Biophys Res Commun, 2001, 288: 420-426

36 Mollerup J, Krogh T N, Nielsen P F, et al. Properties of the co-chaperone protein p23 erroneously attributed to ALG-2 (apoptosis-linked gene 2). FEBS Lett, 2003, 555: 478-482

37 la Cour J M, Mollerup J, Winding P, et al. Up-regulation of ALG-2 in hepatomas and lung cancer tissue. Am J Pathol, 2003, 163: 81-89

38 Hwang I S, Jung Y S, Kim E. Interaction of ALG-2 with ASK1 influences ASK1 localization and subsequent JNK activation. FEBS Lett, 2002, 529: 183-187

39 Chen C, Sytkowski A J. Apoptosis-linked gene-2 connects the Raf-1 and ASK1 signalings. Biochem Biophys Res Commun, 2005, 333: 51-57

40 Vergarajauregui S, Martina J A, Puertollano R. Identification of the penta-EF-hand protein ALG-2 as a $\mathrm{Ca}^{2+}$-dependent interactor of mucolipin-1. J Biol Chem, 2009, 284: 36357-36366

41 Shibata H, Yamada K, Mizuno T, et al. The penta-EF-hand protein ALG-2 interacts with a region containing PxY repeats in Alix/AIP1, which is required for the subcellular punctate distribution of the amino-terminal truncation form of Alix/AIP1. J Biochem, 2004, 135: 117-128

42 Shibata H, Suzuki H, Kakiuchi T, et al. Identification of Alix-type and Non-Alix-type ALG-2-binding sites in human phospholipid scramblase 3: differential binding to an alternatively spliced isoform and amino acid-substituted mutants. J Biol Chem, 2008, 283: 9623-9632

43 Shibata $\mathrm{H}$, Inuzuka $\mathrm{T}$, Yoshida $\mathrm{H}$, et al. The ALG-2 binding site in Sec31A influences the retention kinetics of Sec31A at the endoplasmic reticulum exit sites as revealed by live-cell time-lapse imaging. Biosci Biotechnol Biochem, 2010a, 74: 1819-1826

44 Shibata H, Suzuki H, Yoshida H, et al. ALG-2 directly binds Sec31A and localizes at endoplasmic reticulum exit sites in a $\mathrm{Ca}^{2+}$-dependent manner. Biochem Biophys Res Commun, 2007, 353: 756-763

45 Katoh K, Suzuki H, Terasawa Y, et al. The penta-EF-hand protein ALG-2 interacts directly with the ESCRT-I component TSG101, and $\mathrm{Ca}^{2+}$-dependently co-localizes to aberrant endosomes with dominant-negative AAA ATPase SKD1/Vps4B. Biochem J, 2005, 391: 
$677-685$

46 Draeby I, Woods Y L, la Cour J M, et al. The calcium binding protein ALG-2 binds and stabilizes Scotin, a p53-inducible gene product localized at the endoplasmic reticulum membrane. Arch Biochem Biophys, 2007, 467: 87-94

47 Montaville P, Dai Y, Cheung C Y, et al. Nuclear translocation of the calcium-binding protein ALG-2 induced by the RNA-binding protein RBM22. Biochim Biophys Acta, 2006, 1763: 1335-1343

48 Inuzuka T, Suzuki H, Kawasaki M, et al. Molecular basis for defect in Alix-binding by alternatively spliced isoform of ALG-2 (ALG2DeltaGF122) and structural roles of F122 in target recognition. BMC Struct Biol, 2010, 10: 25

49 Suzuki H, Kawasaki M, Inuzuka $\mathrm{T}$, et al. The mechanism of $\mathrm{Ca}^{2+}$-dependent recognition of Alix by ALG-2: insights from X-ray crystal structures. Biochem Soc Trans, 2009, 37: 190-194

50 Kay B K, Williamson M P, Sudol M. The importance of being proline: the interaction of proline-rich motifs in signaling proteins with their cognate domains. FASEB J, 2000, 14: 231-241

51 Raiborg C, Stenmark H. The ESCRT machinery in endosomal sorting of ubiquitylated membrane proteins. Nature, 2009, 458: 445-452

52 Hurley J H, Hanson P I. Membrane budding and scission by the ESCRT machinery: it's all in the neck. Nat Rev Mol Cell Biol, 2010, 11: 556-566

53 Morita E, Sundquist W I. Retrovirus budding. Annu Rev Cell Dev Biol, 2004, 20: 395-425

54 Morita E, Sandrin V, Chung H Y, et al. Human ESCRT and ALIX proteins interact with proteins of the midbody and function in cytokinesis. EMBO J, 2007, 26: 4215-4227

55 Katoh K, Shibata H, Suzuki H, et al. The ALG-2-interacting protein Alix associates with CHMP4b, a human homologue of yeast Snf7 that is involved in multivesicular body sorting. J Biol Chem, 2003, 278: 39104-39113

56 Garrus J E, von Schwedler U K, Pornillos O W, et al. Tsg101 and the vacuolar protein sorting pathway are essential for HIV-1 budding. Cell, 2001, 107: 55-65

57 von Schwedler U K, Stuchell M, Müller B, et al. The protein network of HIV budding. Cell, 2003, 114: 701-713

58 Ichioka F, Takaya E, Suzuki H, et al. HD-PTP and Alix share some membrane-traffic related proteins that interact with their Brol domains or proline-rich regions. Arch Biochem Biophys, 2007, 457: 142-149

59 Okumura M, Ichioka F, Kobayashi R, et al. Penta-EF-hand protein ALG-2 functions as a $\mathrm{Ca}^{2+}$-dependent adaptor that bridges Alix and TSG101. Biochem Biophys Res Commun, 2009, 386: 237-241

60 Yamasaki A, Tani K, Yamamoto A, et al. The $\mathrm{Ca}^{2+}$-binding protein
ALG-2 is recruited to endoplasmic reticulum exit sites by Sec31A and stabilizes the localization of Sec31A. Mol Biol Cell, 2006, 17: 4876-4887

61 la Cour J M, Mollerup J, Berchtold M W. ALG-2 oscillates in subcellular localization, unitemporally with calcium oscillations. Biochem Biophys Res Commun, 2007, 353: 1063-1067

62 Bentley M, Nycz D C, Joglekar A, et al. Vesicular calcium regulates coat retention, fusogenicity, and size of pre-Golgi intermediates. Mol Biol Cell, 2010, 21: 1033-1046

63 Shibata H, Sugiura H, Yokoyama T, et al. Recruitment of annexin A11 to endoplasmic reticulum exit sites is mediated by the adaptor function of the penta-EF-hand protein ALG-2. Acta Biochim Pol, 2010b, 57: 27

64 Jang I K, Hu R, Lacaná E, et al. Apoptosis-linked gene 2-deficient mice exhibit normal T-cell development and function. Mol Cell Biol, 2002, 22: 4094-4100

65 Rao R V, Poksay K S, Castro-Obregon S, et al. Molecular components of a cell death pathway activated by endoplasmic reticulum stress. J Biol Chem, 2004, 279: 177-187

66 Mahul-Mellier A L, Hemming F J, Blot B, et al. Alix, making a link between apoptosis-linked gene-2, the endosomal sorting complexes required for transport, and neuronal death in vivo. J Neurosci, 2006, 26: 542-549

67 Mahul-Mellier A L, Strappazzon F, Petiot A, et al. Alix and ALG-2 are involved in tumor necrosis factor receptor 1-induced cell death. J Biol Chem, 2008, 283: 34954-34965

68 Høj B R, la Cour J M, Mollerup J, et al. ALG-2 knockdown in HeLa cells results in $\mathrm{G} 2 / \mathrm{M}$ cell cycle phase accumulation and cell death. Biochem Biophys Res Commun, 2009, 378: 145-148

69 la Cour J M, Høj B R, Mollerup J, et al. The apoptosis linked gene ALG-2 is dysregulated in tumors of various origin and contributes to cancer cell viability. Mol Oncol, 2008, 1: 431-439

70 Aviel-Ronen S, Coe B P, Lau S K, et al. Genomic markers for malignant progression in pulmonary adenocarcinoma with bronchioloalveolar features. Proc Natl Acad Sci USA, 2008, 105: 1015510160

71 Yamada Y, Arao T, Gotoda T, et al. Identification of prognostic biomarkers in gastric cancer using endoscopic biopsy samples. Cancer Sci, 2008, 99: 2193-2199

72 Yap K L, Ames J B, Swindells M B, et al. Diversity of conformational states and changes within the EF-hand protein superfamily. Proteins, 1999, 37: 499-507

73 Crivici A, Ikura M. Molecular and structural basis of target recognition by calmodulin. Ann Rev Biophys Biomol Struct, 1995, 24: 85-116

Open Access This article is distributed under the terms of the Creative Commons Attribution License which permits any use, distribution, and reproduction in any medium, provided the original author(s) and source are credited. 